\title{
ASYMPTOTIC PROPERTIES OF LINEAR FOURTH ORDER DIFFERENTIAL EQUATIONS
}

\author{
SHAIR AHMAD
}

ABSTRACT. Asymptotic behavior of solutions is studied for the general fourth order linear homogeneous differential equation with bounded coefficients. It is shown that roughly the same qualitative behavior as in the constant coefficient case holds.

1. Introduction. Consider the general fourth order linear homogeneous differential equation written in the form

$$
u^{(4)}=b_{3}(t) u^{\prime \prime \prime}+b_{2}(t) u^{\prime \prime}+b_{1}(t) u^{\prime}+b_{0}(t) u .
$$

If $b_{k} \in C[a, \infty), k=0,1,2$, and $b_{3} \in C^{3}[a, \infty)$, the substitution

$$
u(t)=y(t) \exp \left(\frac{1}{4} \int_{a}^{t} b_{3}(s) d s\right)
$$

simplifies this differential equation to the form

$$
y^{(4)}=p(t) y^{\prime \prime}+q(t) y^{\prime}+r(t) y
$$

with $p, q$ and $r$ continuous on $[a, \infty)$. In this paper we study the asymptotic behavior of a class of differential equations of the form (L).

Our motivation comes from the following two observations:

1. If $p, q$, and $r$ are constants with $p \geqslant 0, q>0$, and $r>0$ and $S$ denotes the vector space of solutions of $(\mathrm{L})$ which together with their derivatives tend to zero as $t \rightarrow+\infty$, then $S$ has dimension three. If $y$ is any solution of $(\mathrm{L})$ not in $S$, then $\left|y^{(k)}(t)\right| \rightarrow+\infty$ as $t \rightarrow+\infty, k=0,1,2, \ldots$

2. If $p, q$, and $r$ are constants with $p \geqslant 0, q<0$, and $r>0$, then $S$ has dimension one. Any solution of (L) not in $S$ cannot be bounded.

These observations follow easily by studying the location of the zeros of the characteristic polynomial $\lambda^{4}-p \lambda^{2}-q \lambda-r$.

The object of this paper is to show that the solutions of $(\mathrm{L})$ have roughly the same qualitative behavior as in the constant coefficient cases (1) and (2), respectively, under the following two sets of conditions:

$\mathrm{C}_{1}: p, q, r, p^{\prime}, r^{\prime}$ continuous on the half-axis $[a, \infty), p(t) \geqslant 0, r(t) \geqslant m>0$, $r^{\prime}(t) \leqslant 0, p^{\prime}(t)-2 q(t) \leqslant-d<0 ; p$ and $q$ bounded.

Received by the editors May 19, 1975.

AMS (MOS) subject classifications (1970). Primary 34A30; Secondary 34D05.

Key words and phrases. Differential equation, linear, bounded, dimension, asymptotic. 
$\mathrm{C}_{2}: p, q, r, p^{\prime}, r^{\prime}$ continuous on $[a, \infty), p(t) \geqslant 0, r(t)>0, r^{\prime}(t) \geqslant 0, p^{\prime}(t)$ $-2 q(t) \geqslant d>0 ; p, q$ and $r$ bounded.

Using techniques of Lazer [4], Singh [5] studied third order linear differential equations with bounded coefficients. Similarly, the author [1] studied a simple selfadjoint differential equation of order four. The results in this paper are mainly motivated by [4] and [5].

2. First we state three almost obvious facts in the form of lemmas. The proofs of these lemmas are elementary and trivial.

Lemma 1. Let $f \in C^{1}[a, \infty)$. If $\int_{a}^{\infty} f^{2}(t) d t<+\infty$ and $f^{\prime}(t)$ is bounded, then $f(t) \rightarrow 0$ as $t \rightarrow \infty$.

Lemma 2. Let $f \in C^{2}[a, \infty)$. If $f(t) \rightarrow 0$ as $t \rightarrow \infty$ and $f^{\prime \prime}(t)$ is bounded, then $f^{\prime}(t) \rightarrow 0$ as $t \rightarrow \infty$.

LEMma 3. If $f \in C^{2}[a, \infty)$ and $f(t)$ and $f^{\prime \prime}(t)$ are bounded, then $f^{\prime}(t)$ is bounded.

In this section we consider the behavior of solutions of (L) subject to the conditions $\mathrm{C}_{1}$. If $y(t)$ is a solution of $(\mathrm{L})$ and

$$
F[y(t)] \equiv r(t) y(t)^{2}-2 y^{\prime \prime \prime}(t) y^{\prime}(t)+y^{\prime \prime}(t)^{2}+p(t) y^{\prime}(t)^{2},
$$

then, as may be verified by differentiation,

$$
F[y(t)]=F[y(a)]+\int_{a}^{t} r^{\prime}(s) y(s)^{2} d s-\int_{a}^{t}\left[2 q(s)-p^{\prime}(s)\right] y^{\prime}(s)^{2} d s .
$$

Under the conditions $\mathrm{C}_{1}$ it is easy to see that if $y(t)$ is any nontrivial solution of $(\mathrm{L}), y^{\prime}(t)$ cannot be identically equal to zero in any interval. Hence, the conditions $\mathrm{C}_{1}$ imply that for any nontrivial solution $y(t)$ of $(\mathrm{L}), F[y(t)]$ is strictly decreasing.

LEMMA 4. Let the conditions $\mathrm{C}_{1}$ hold. If $y(t)$ is a solution of (L) for which $F[y(t)] \geqslant 0$ for all $t \in[a, \infty)$ then $y^{(k)}(t) \rightarrow 0$ as $t \rightarrow \infty, k=0,1,2,3,4$.

Proof. Since $r^{\prime}(t) \leqslant 0$ and $2 q(t)-p^{\prime}(t) \geqslant d>0$, it follows from (1) that for all $t \geqslant a, \int_{a}^{t} y^{\prime}(s)^{2} d s \leqslant F[y(a)] / d$ and hence

$$
\int_{a}^{\infty} y^{\prime}(s)^{2} d s<+\infty
$$

We assert that $y^{\prime \prime}(t)$ is bounded. To see this, consider the two possibilities: (i) $y^{\prime \prime \prime}(t)$ vanishes for arbitrarily large values of $t$; (ii) $y^{\prime \prime \prime}(t) \neq 0$ for $t \geqslant b \geqslant a$. If (i) holds and $x$ is a zero of $y^{\prime \prime \prime}(t)$ then

$$
y^{\prime \prime}(x)^{2} \leqslant r(x) y(x)^{2}+y^{\prime \prime}(x)^{2}+p(x) y^{\prime}(x)^{2}=F[y(x)] \leqslant F[y(a)] .
$$

Thus if (i) holds, $y^{\prime \prime}(t)$ is bounded on the set of zeros of $y^{\prime \prime \prime}(t)$ and hence bounded on $[a, \infty)$. If (ii) holds, there exists $c>b$ such that $y^{\prime \prime \prime}(t) y^{\prime \prime}(t) \neq 0$ for $t \geqslant c$. The inequality $y^{\prime \prime \prime}(t) y^{\prime \prime}(t)>0$ for $t \geqslant c$ implies $\lim _{t \rightarrow \infty} y^{\prime}(t)= \pm \infty$, contrary to (2), thus $y^{\prime \prime \prime}(t) y^{\prime \prime}(t)<0$ for $t \geqslant c$. Hence, if (ii) holds, $y^{\prime \prime}(t)$ approaches a finite limit as $t \rightarrow \infty$ and is therefore bounded on $[a, \infty)$. 
From the boundedness of $y^{\prime \prime}(t)$, Lemma 1 and (2) we conclude that

$$
\lim _{t \rightarrow \infty} y^{\prime}(t)=0 \text {. }
$$

We assert that $y(t)$ must be bounded on $[a, \infty)$. To see this consider the two possibilities: (i) $y^{\prime}(t)$ vanishes for arbitrarily large values of $t$; (ii) $y^{\prime}(t) \neq 0$ for $t \geqslant b \geqslant a$. If (i) holds and $x$ is a zero of $y^{\prime}(t)$ then, since $r(x) \geqslant m>0$,

$$
y(x)^{2} \leqslant m^{-1}\left[r(x) y(x)^{2}+y^{\prime \prime}(x)^{2}\right]=m^{-1} F[y(x)] \leqslant m^{-1} F[y(a)] .
$$

Thus, if (i) holds, $y(t)$ is bounded on the set of zeros of $y^{\prime}(t)$ and hence bounded on $[a, \infty)$. If (ii) holds, $y(t)$ must tend to a finite limit or $\pm \infty$ as $t \rightarrow \infty$. But since $p, q, y^{\prime}$ and $y^{\prime \prime}$ are bounded and $r(t) \geqslant m>0$ the equality

$$
y^{(4)}=p(t) y^{\prime \prime}+q(t) y^{\prime}+r(t) y
$$

implies that $\lim _{t \rightarrow \infty} y(t)$ must be finite so that (ii) implies the boundedness of $y(t)$.

From the boundedness of $y, y^{\prime}, y^{\prime \prime}, p, q$, and $r$ it follows that $y^{(4)}(t)$ is bounded on $[a, \infty)$, and so by Lemma $3, y^{\prime \prime \prime}(t)$ is bounded on $[a, \infty)$. From (3) and Lemma 2 applied to $y^{\prime}(t)$ it follows that

$$
\lim _{t \rightarrow \infty} y^{\prime \prime}(t)=0 \text {. }
$$

The boundedness of $y^{(4)}(t)$ and (5) together with Lemma 2 imply that

$$
\lim _{t \rightarrow \infty} y^{\prime \prime \prime}(t)=0 \text {. }
$$

It remains to show that $y(t) \rightarrow 0$ as $t \rightarrow \infty$. To this end we consider $F[y(t)]$. Since $F[y(t)]$ is nonincreasing and nonnegative, $\lim _{t \rightarrow \infty} F[y(t)]$ exists. Thus (3), (5), (6) and the boundedness of $p(t)$ implies

$$
\begin{aligned}
\lim _{t \rightarrow \infty} F[y(t)] & =\lim _{t \rightarrow \infty}\left(r(t) y(t)^{2}-y^{\prime \prime \prime}(t) y^{\prime}(t)+y^{\prime \prime}(t)^{2}+p(t) y^{\prime}(t)^{2}\right) \\
& =\lim _{t \rightarrow \infty}\left(r(t) y^{2}(t)\right) .
\end{aligned}
$$

Thus, since $r(t) \geqslant m>0$ and $r^{\prime}(t) \leqslant 0, \lim _{t \rightarrow \infty} y(t)$ exists. If this limit were nonzero, (3), (4), (5), and the boundedness of $p$ and $q$ would imply that $\lim _{t \rightarrow \infty} y^{(4)}(t)$ exists and is nonzero, contradicting (6). Hence $\lim _{t \rightarrow \infty} y(t)=0$ and the proof of Lemma 4 is complete.

LEMMA 5. Let the conditions $\mathrm{C}_{1}$ hold. If $z(t)$ is a solution of $(\mathrm{L})$ satisfying the initial conditions $z(a)=0, z^{\prime}(a)=0, z^{\prime \prime}(a)=0, z^{\prime \prime \prime}(a)>0$, then $\lim _{t \rightarrow \infty} z(t)$ $=+\infty$ and $\lim _{t \rightarrow \infty} z^{\prime}(t)=+\infty$.

Proof. Since $F[z(a)]=0$ and $F[z(t)]$ is strictly decreasing,

$$
F[z(t)]=r(t) z(t)^{2}-2 z^{\prime \prime \prime}(t) z^{\prime}(t)+z^{\prime \prime}(t)^{2}+p(t) z^{\prime}(t)^{2}<0
$$

for $t>a$. Hence $z^{\prime \prime \prime}(t) z^{\prime}(t)>0$ for $t>a$ and so $z^{\prime \prime \prime}(t)>0$ for $t>a$. From this the conclusion of the lemma follows immediately. 
THEOREM 1. Let the conditions $\mathrm{C}_{1}$ hold. Let $S$ denote the vector space of solutions of $(\mathrm{L})$ which tend to zero with their first four derivatives. $S$ has dimension three, and if $y(t)$ is any solution of $(\mathrm{L})$ not in $S$ then $\lim _{t \rightarrow \infty}|y(t)|=\lim _{t \rightarrow \infty}\left|y^{\prime}(t)\right|$ $=\infty$.

Proof. Let $z_{0}, z_{1}, z_{2}, z_{3}$ denote the solutions of (L) satisfying the initial conditions

$$
z_{j}^{(k)}(a)=\delta_{k j}=\left\{\begin{array}{l}
0, k \neq j, \\
1, \quad k=j,
\end{array} j, k=0,1,2,3 .\right.
$$

For each positive integer $n>a$, let $a_{0 n}, a_{3 n}, b_{1 n}, b_{3 n}, c_{2 n}$ and $c_{3 n}$ be numbers such that

$$
\begin{gathered}
a_{0 n} z_{0}^{\prime}(n)+a_{3 n} z_{3}^{\prime}(n)=0, \quad b_{1 n} z_{1}^{\prime}(n)+b_{3 n} z_{3}^{\prime}(n)=0, \\
c_{2 n} z_{2}^{\prime}(n)+c_{3 n} z_{3}^{\prime}(n)=0,
\end{gathered}
$$

and

$$
a_{0 n}^{2}+a_{3 n}^{2}=b_{1 n}^{2}+b_{3 n}^{2}=c_{2 n}^{2}+c_{3 n}^{2}=1 .
$$

Let $u_{n}(t), v_{n}(t)$ and $w_{n}(t)$ be the nontrivial solutions of (L) defined by $u_{n}=a_{0 n} z_{0}+a_{3 n} z_{3}, v_{n}=b_{1 n} z_{1}+b_{3 n} z_{3}, w_{n}=c_{2 n} z_{2}+c_{3 n} z_{3}$. Since $F\left[u_{n}(t)\right]$, $F\left[v_{n}(t)\right]$, and $F\left[w_{n}(t)\right]$ are strictly decreasing on $[a, \infty)$ and $F\left[u_{n}(n)\right] \geqslant 0$, $F\left[v_{n}(n)\right] \geqslant 0$, and $F\left[w_{n}(n)\right] \geqslant 0$, it follows that

$$
F\left[u_{n}(t)\right]>0, \quad F\left[v_{n}(t)\right]>0, \quad F\left[w_{n}(t)\right]>0 \text { for all } t \in[a, n) .
$$

From (7) there exists a sequence of integers $\left\{n_{k}\right\}$ and numbers $a_{0}, a_{3}, b_{1}, b_{3}$, $c_{2}$ and $c_{3}$ such that $a_{0 n_{k}} \rightarrow a_{0}, a_{3 n_{k}} \rightarrow a_{3}, b_{1 n_{k}} \rightarrow b_{1}, b_{3 n_{k}} \rightarrow b_{3}, c_{2 n_{k}} \rightarrow c_{2}, c_{3 n_{k}}$ $\rightarrow c_{3}$ as $n_{k} \rightarrow \infty$. Clearly,

$$
a_{0}^{2}+a_{3}^{2}=b_{1}^{2}+b_{3}^{2}=c_{2}^{2}+c_{3}^{2}=1 .
$$

Let $u(t), v(t)$ and $w(t)$ be the solutions of (L) defined by

$$
u=a_{0} z_{0}+a_{3} z_{3}, \quad v=b_{1} z_{1}+b_{3} z_{3}, \quad w=c_{2} z_{2}+c_{3} z_{3} .
$$

By the linear independence of $z_{0}, z_{1}, z_{2}$ and $z_{3}$ and (9) it follows that $u, v$, and $w$ are nontrivial solutions of (L). Clearly, $u_{n_{k}}^{(j)}(t) \rightarrow u^{(j)}(t), j=0,1,2,3$, as $n_{k} \rightarrow \infty$, thus $F\left[u_{n_{k}}(t)\right] \rightarrow F[u(t)]$ as $n_{k} \rightarrow \infty$. Thus, by (8), $F[u(t)] \geqslant 0$ for all $t \geqslant a$ and similar reasoning shows that $F[v(t)] \geqslant 0$ and $F[w(t)] \geqslant 0$ for all $t \geqslant a$. Hence by Lemma $4, u, v$ and $w$ are members of $S$. If $u, v$, and $w$ were linearly dependent there would exist constants $k_{1}, k_{2}$, and $k_{3}$, not all zero, such that $k_{1} u(t)+k_{2} v(t)+k_{3} w(t) \equiv 0$. Hence by (10) and the independence of $z_{0}$, $z_{1}, z_{2}$ and $z_{3}$ it would follow that $0=k_{1} a_{0}=k_{2} b_{1}=k_{3} c_{2}$. If $k_{1} \neq 0$ then $u(t)= \pm z_{3}(t)$, hence by Lemma $5, \lim _{t \rightarrow \infty}|u(t)|=\infty$, contradicting the fact that $u \in S$. Similarly the assumptions $k_{2} \neq 0$ and $k_{3} \neq 0$ lead to contradictions. Hence $u, v$, and $w$ are independent and the dimension of $S$ is at least three. 
Consider the solutions $u, v, w$, and $z_{3}$. If for some constants $a, b, c$, and $d$, not all zero, $a u(t)+b v(t)+c w(t)+d z_{3}(t) \equiv 0$ then by the independence of $u$, $v$, and $w, d \neq 0$ which implies $z_{3} \in S$, contradicting Lemma 5 . Hence $u, v, w$, and $z_{3}$ are independent, and since the order of (L) is four, it follows from the theory of linear differential equations that for any solution $y$ of (L), $y$ $=a u+b v+c w+d z_{3}$ for some constants $a, b, c$ and $d$. Since $u, v$, and $w$ are in $S$ and $\lim _{t \rightarrow \infty}\left|z_{3}(t)\right|=+\infty, y \in S$ if and only if $d=0$. Hence the dimension of $S$ is exactly three and for any solution $y(t)$ of (L) not in $S$ both $|y(t)|$ and $\left|y^{\prime}(t)\right|$ tend to infinity as $t$ tends to infinity.

3. We will now consider the behavior of the solutions of (L) subject to the conditions $\mathrm{C}_{2}$. Under these conditions if $y(t)$ is any nontrivial solution of $(\mathrm{L})$, $y^{\prime}(t)$ cannot be identically equal to zero in any interval. Moreover the conditions $r^{\prime}(t) \geqslant 0, p^{\prime}(t)-2 q(t) \geqslant d>0$ and the identity

$$
F[y(t)]=F[y(a)]+\int_{a}^{t} r^{\prime}(s) y^{2}(s) d s+\int_{a}^{t}\left[p^{\prime}(s)-2 q(s)\right] y^{\prime}(s)^{2} d s
$$

imply that if the conditions $\mathrm{C}_{2}$ hold then for any nontrivial solution $y(t)$ of $(\mathrm{L})$ the function $F[y(t)]$ is strictly increasing.

THEOREM 2. Let the conditions $\mathrm{C}_{2}$ hold. There exists a solution $w(t)$ of $(\mathrm{L})$ with the properties $w(t)>0, w^{\prime}(t)<0, w^{\prime \prime}(t)>0, w^{\prime \prime \prime}(t)<0$ for all $t \in[a, \infty)$ and $\lim _{t \rightarrow \infty} w(t)=\lim _{t \rightarrow \infty} w^{\prime}(t)=\lim _{t \rightarrow \infty} w^{\prime \prime}(t)=0$.

PROOF. Let $z_{0}(t), z_{1}(t), z_{2}(t)$ and $z_{3}(t)$ denote the solutions of (L) introduced in the proof of Theorem 1. For each integer $n>a$ it follows from elementary algebra that there exist numbers $c_{0 n}, c_{1 n}, c_{2 n}$ and $c_{3 n}$ such that

$$
\begin{aligned}
c_{0 n} z_{0}(n)+c_{1 n} z_{1}(n)+c_{2 n} z_{2}(n)+c_{3 n} z_{3}(n) & =0, \\
c_{0 n} z_{0}^{\prime}(n)+c_{1 n} z_{1}^{\prime}(n)+c_{2 n} z_{2}^{\prime}(n)+c_{3 n} z_{3}^{\prime}(n) & =0, \\
c_{0 n} z_{0}^{\prime \prime}(n)+c_{1 n} z_{1}^{\prime \prime}(n)+c_{2 n} z_{2}^{\prime \prime}(n)+c_{3 n} z_{3}^{\prime \prime}(n) & =0, \\
c_{0 n}^{2}+c_{1 n}^{2}+c_{2 n}^{2}+c_{3 n}^{2} & =1 .
\end{aligned}
$$

Let $w_{n}(t)$ be the solution of (L) defined by $w_{n}=c_{0 n} z_{0}+c_{1 n} z_{1}+c_{2 n} z_{2}$ $+c_{3 n} z_{3}$. By the independence of the solutions $z_{0}, z_{1}, z_{2}, z_{3}$ and (11), $w_{n}$ is a nontrivial solution of $(\mathrm{L})$ so that

$$
F\left[w_{n}(t)\right]=r(t) w_{n}^{2}(t)-2 w_{n}^{\prime \prime \prime}(t) w_{n}^{\prime}(t)+w_{n}^{\prime \prime}(t)^{2}+p(t) w_{n}^{\prime}(t)^{2}
$$

is strictly increasing. Thus, since $F\left[w_{n}(n)\right]=0$ it follows that

$$
F\left[w_{n}(t)\right]<0 \text { for } t \in[a, n) .
$$

By (11) there exists a sequence of integers $n_{k}$ and numbers $c_{j}, j=0,1,2,3$, such that $\lim _{n_{k} \rightarrow \infty} c_{j n_{k}}=c_{j}$. Let $w(t)$ be the solution of $(\mathrm{L})$ defined by $w=c_{0} z_{0}+c_{1} z_{1}+c_{2} z_{2}+c_{3} z_{3}$. From the independence of the $z_{j}, j=0,1,2$, 3 , and the equality $c_{0}^{2}+c_{1}^{2}+c_{2}^{2}+c_{3}^{2}=1$, it follows that $w$ is a nontrivial solution of (L) so that $F[w(t)]$ is strictly increasing. Moreover, since for each fixed $t, w_{n_{k}}^{(j)}(t) \rightarrow w^{(j)}(t), j=0,1,2,3$, as $n_{k} \rightarrow \infty, F\left[w_{n_{k}}(t)\right] \rightarrow F[w(t)]$ as $n_{k}$ 
$\rightarrow \infty$, and so by (12)

$$
F[w(t)] \leqslant 0, \quad t \in[a, \infty) .
$$

If for some $x \in[a, \infty), F[w(x)]=0$ then, since $F[w(t)]$ is increasing, it would follow that $F[w(t)]>0$ for $t>x$, contradicting (13). Hence

$$
F[w(t)]=r(t) w(t)^{2}-2 w^{\prime \prime \prime}(t) w^{\prime}(t)+w^{\prime \prime}(t)^{2}+p(t) w^{\prime}(t)^{2}<0
$$

for all $t \in[a, \infty)$. From this we infer (i) $w^{\prime \prime \prime}(t) w^{\prime}(t)>0$ for all $t \in[a, \infty)$; (ii) $\lim _{t \rightarrow \infty} F[w(t)]$ exists. By interchanging the roles of $w(t)$ and $-w(t)$ we can assume

$$
w^{\prime \prime \prime}(t)<0, \quad w^{\prime}(t)<0, \quad t \in[a, \infty) .
$$

From (ii), the conditions $r^{\prime}(t) \geqslant 0, p^{\prime}(t)-2 q(t) \geqslant d>0$ and the identity

$$
F[w(t)]=F[w(a)]+\int_{a}^{t} r^{\prime}(s) w(s)^{2} d s+\int_{a}^{t}\left[p^{\prime}(s)-2 q(s)\right] w^{\prime}(s)^{2} d s
$$

we infer that

$$
\int_{a}^{\infty} w^{\prime}(s)^{2} d s<+\infty
$$

(14) implies that $w^{\prime \prime}(t) \neq 0$ for sufficiently large $t$, and (14) and (15) imply that $w^{\prime \prime}(t)>0$ for sufficiently large $t$. Hence by (14),

$$
w^{\prime \prime}(t)>0 \text { for all } t \in[a, \infty) .
$$

By (14), $w(t) \neq 0$ for large $t$. If $w(t)<0$ for large $t, \lim _{t \rightarrow \infty} w(t)=m<0$ or $\lim _{t \rightarrow \infty} w(t)=-\infty$. But (14) and (16) imply that $w^{\prime \prime}(t)$ and $w^{\prime}(t)$ are bounded and so, if the above condition held, the boundedness of $p(t), q(t)$, Lemma 2, and the conditions $r(t)>0, r^{\prime}(t) \geqslant 0$ would imply that $w^{(4)}=p(t) w^{\prime \prime}$ $+q(t) w^{\prime}+r(t) w(t)<0$ for large $t$ which together with (14) contradicts (16). Hence $w(t)>0$ for large $t$ and so by (14), $w(t)>0$ for all $t \in[a, \infty)$. Thus $\lim _{t \rightarrow \infty} w(t)=c \geqslant 0$. If $c>0, w^{(4)}(t)>\alpha>0$ from a certain point on, contradicting (14). Hence $\lim _{t \rightarrow \infty} w(t)=0$ and similarly $\lim _{t \rightarrow \infty} w^{\prime}(t)$ $=1$ in proves the theorem.

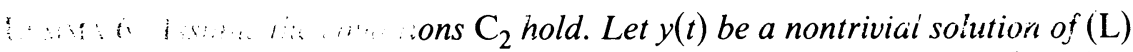
for $\quad$ some $x \in[a, \infty)$. $y(t)$ cannot be bounded on $[a, \infty)$.

Proof. Although an elementary proof based on Lemmas 1, 2, and 3 can be given, for brevity we will rely on the following nontrivial result due to $\mathrm{E}$. Esclangon [3] (for another source see [2]): Let the functions $p_{i}(t), i=0,1, \ldots$, $n$, be continuous and bounded for $t \geqslant t_{0}$. If $y^{(n)}+p_{1}(t) y^{(n-1)}+\cdots+p_{n}(t) y$ $=p_{0}(t)$ and $y(t)$ is bounded for $t \geqslant t_{0}$, then its derivatives $y^{(k)}(1 \leqslant k \leqslant n)$ are also bounded for $t \geqslant t_{0}$.

To prove the lemma we shall assume that $y(t)$ is a nontrivial bounded solution of (L) with $F\left[y\left(t_{0}\right)\right] \geqslant 0, t_{0} \geqslant a$ and arrive at a contradiction. Since

$$
F[y(t)]=r(t) y(t)^{2}-2 y^{\prime \prime \prime}(t) y^{\prime}(t)+y^{\prime \prime}(t)^{2}+p(t) y^{\prime}(t)^{2}
$$


is strictly increasing and since Esclangon's theorem and the boundedness of $p$ and $r$ imply the boundedness of $F[y(t)], \lim _{t \rightarrow \infty} F[y(t)]$ exists and is equal to a positive number $\alpha$. Hence for $t \geqslant a$,

$$
\begin{aligned}
\int_{a}^{t} y^{\prime}(s)^{2} d s & \leqslant \frac{1}{d}\left[\int_{a}^{t}\left[p^{\prime}(s)-2 q(s)\right] y^{\prime}(s) d s+\int_{a}^{t} r^{\prime}(s) y(s)^{2} d s+F[y(a)]\right] \\
& =d^{-1} F[y(t)]<\alpha / d,
\end{aligned}
$$

and so $\int_{0}^{\infty} y^{\prime}(t)^{2} d t<+\infty$. Since $y^{\prime \prime}(t)$ is bounded it follows from Lemma 1 that $\lim _{t \rightarrow \infty} y^{\prime}(t)=0$. The boundedness of $y^{\prime \prime \prime}(t)$ and $y^{(4)}(t)$ and Lemma 2 imply that $\lim _{t \rightarrow \infty} y^{\prime \prime}(t)=0$ and $\lim _{t \rightarrow \infty} y^{\prime \prime \prime}(t)=0$. Thus $\lim _{t \rightarrow \infty} F[y(t)]$ $=\lim _{t \rightarrow \infty} r(t) y(t)^{2}$. But $r(t)>0, r^{\prime}(t) \geqslant 0$ and $r(t)$ is bounded so that $\lim _{t \rightarrow \infty} y(t)=\delta \neq 0$. Hence $\lim _{t \rightarrow \infty} y^{(4)}(t)=\gamma \neq 0$ which contradicts $\lim _{t \rightarrow \infty} y^{\prime \prime \prime}(t)=0$. This contradiction proves the lemma.

THEOREM 3. Let the conditions $\mathrm{C}_{2}$ hold. If $z(t)$ is a bounded solution of $(\mathrm{L})$ then for some constant $k, z(t)=k w(t)$ where $w(t)$ is the solution of $(\mathrm{L})$ whose existence was established in Theorem 2.

Proof. Let $z(t)$ be a bounded solution of (L) and let $k$ be a number such that $z^{\prime}(a)-k w^{\prime}(a)=0$. Let $y(t)=z(t)-k w(t)$. If $y(t)$ were not identically zero then, since $F[y(a)] \geqslant 0$, it would follow from Lemma 6 that $y(t)$ could not be bounded, contradicting the boundedness of $w(t)$ and $z(t)$. This contradiction proves the theorem.

\section{REFERENCES}

1. Shair Ahmad, On the oscillation of solutions of a class of linear fourth order differential equations, Pacific J. Math. 34 (1970), 289-299. MR 42 \#3352.

2. W. A. Coppel, Stability and asymptotic behavior of differential equations, Heath, Boston, Mass., 1965. MR 32 \# 7875.

3. E. Esclangon, Nouvelles recherches sur les functions quasi-périodiques, Ann. Obs., Bordeaux 16 (1917), 15-226.

4. A. C. Lazer, The behavior of solutions of the differential equation $y^{\prime \prime \prime}+p(x) y^{\prime}+q(x) y=0$, Pacific J. Math. 17 (1966), 435-466. MR 33 \# 1552.

5. Y. P. Singh, The asymptotic behavior of solutions of linear third order differential equations, Proc. Amer. Math. Soc. 20 (1969), 309-314.

Department of Mathematics, Oklahoma State University, Stillwater, OKlahoma 74074 